\title{
A Mathematical Model of Biofilm Growth on Degradable Substratum
}

\author{
Jack M. Hughes* \\ Sponsor: Hermann J. Eberl ${ }^{\dagger}$ \\ Department of Mathematics and Statistics, University of Guelph \\ Guelph, Ontario, Canada N1G2W1
}

April 12, 2020

\begin{abstract}
We derive a mathematical model for biofilm growth on a degradable substratum. The starting point is the one-dimensional Wanner-Gujer biofilm model. The processes included are diffusion of a dissolved growth controlling substrate into the biofilm, cell lysis, detachment of biomass, and growth from substrate conversion and substratum degradation. The resulting model is a system of two non-linear ordinary differential equations, the evaluation of the right-hand side of which requires the solution of a semi-linear two-point boundary value problem. We perform standard analysis from the existence and uniqueness of solutions to equilibrium and stability analysis. Finally, we study our model through numerical simulations and investigate how biofilms evolve on degradable substratum. We find in our setting that the addition of substratum degradation has no effect on the long-term behaviour of the biofilm, but does affect the transient behaviour.
\end{abstract}

Keywords: Biofilm, Degradable substratum, Mathematical model.

\section{Introduction}

A large proportion of the planet's microbial biomass reside in compact, sessile communities called bacterial biofilms $[2,5,9]$. These biofilms attach to biotic or abiotic surfaces called substratum. When the bacteria attach to the substratum, they become sessile and form a gel-like matrix of extracellular polymeric substances (EPS), in which the bacteria are themselves embedded $[8,13]$. This EPS provides both mechanical protection and protection against antimicrobials. This complex structure allows biofilms to adapt to many environments, including living tissue, implanted medical devices, aquatic systems, and food processing plants $[2,9]$.

Biofilm based technologies have been developed for environmental engineering applications for many decades, originally in wastewater treatment, and more recently in soil

\footnotetext{
*jhughe12@uoguelph.ca

†heberl@uoguelph.ca
} 
remediation and groundwater protection [2]. Other occurrences of biofilms are generally negative. For example, in food processing equipment, biofilms pose a public health risk, which in some circumstances may lead to bacterial infections. In a medical context, biofilms can lead to bacterial infections. In industrial equipment, biofilms can lead to biocorrosion or biofouling, which reduces the overall efficiency of the machinery.

Biofilms are spatially structured microbial populations. Substrates that are necessary to sustain bacterial growth diffuse through the biofilm/EPS matrix, generally from the bulk substrate. As the substrate diffuses through the biofilm, the bacteria consume the substrate, which causes different living conditions for the bacteria inside the biofilm. Specifically, the bacteria at the biofilm/bulk aqueous phase layer have plenty of nutrients, while the bacteria at the biofilm/substratum interface have limited access to the substrate. Hence, variations in metabolic activities within the biofilm occur.

Biofilm detachment is a complex process with many complex mechanisms. The detachment process mediates the accumulation of biofilms and affects the long-term behaviour of the biofilm [2]. Detachment also contributes to the production of suspended flocs [5]. However, we assume that when biomass detaches from the biofilm, it immediately washes out. Throughout the biofilm modelling literature, there are many different detachment rates proposed with their effects on biofilm growth and formation investigated. Common detachment expressions include a constant detachment rate, detachment proportional to the biofilm thickness, and shear dependent detachment rates. We limit ourselves to detachment rate functions that satisfy certain properties, outlined in Section 2. Our prescribed properties permit the constant and proportional detachment rates mentioned above, along with two shear dependent detachment expressions outlined in [1].

Typically, models in the literature assume that the substratum on which the biofilms grow is inert $[1,2,5,7,9]$, which is the case for most biofilm systems. However, we will be investigating biofilm growth on a degradable substratum. This phenomenon occurs in many different environmental engineering applications, including soil remediation and bioenergy/bioethanol production [10], which uses bacteria to degrade organic material, which produces ethanol. Therefore, there is a need to investigate the effects of substratum degradation on biofilm growth.

We start by using the one-dimensional Wanner-Gujer model [14], which has become the basis for most biofilm engineering applications over the past few decades [13]. The model consists of a mass balance based on conservation principles. The underlying assumptions in the model are (i) the biofilm covers the substratum in a homogeneous layer, (ii) the properties of the biofilm change only in the direction perpendicular to the substratum, (iii) the biomass density inside the biofilm is constant; therefore, biomass production corresponds 1:1 to biofilm growth, (iv) the flux of substrate into the biofilm follows Fick's first law [14]. These substrate fluxes are usually computed based on a steady-state assumption, which depends on the disparity of time scales between processes inside the biofilm and biofilm growth [13]. From here, we add the notion of biomass detachment and substratum degradation, where we assume the degradation promotes bacterial growth.

The notion of substratum degradation that we use in our model is a first look into how substratum degradation may affect biofilm dynamics in a specific system. The notion is not based on any biological data, but is derived from qualitative phenomenological assumptions, which appears the simplest conceivable scenario and therefore is suitable as a starting point for the modelling of substratum degradation by biofilms. We assume that substratum degradation occurs whenever the bacteria inside the biofilm at the biofilmsubstratum interface layer are active. For our model, this requires a sufficiently large 
amount of substrate inside the biofilm at the interface layer for substratum degradation to occur. As an example, this notion of substratum degradation reflects a multi-species biofilm system where one bacteria favours the bulk substrate and the other favours the nutrients produced by degrading the substratum. We see that in these systems, the bacteria which favour the substratum would need to be active to degrade the substratum. Multi-species biofilm models are very complicated and are very computationally intensive to simulate. Therefore, as this is the first look into the effects of substratum degradation on biofilm growth, we limit ourselves to a single-species biofilm.

The resulting model is a system of two first-order non-linear ordinary differential equations (ODEs) coupled with a second-order semi-linear boundary value problem (BVP). The system of ODEs describes the change in biofilm and substratum thicknesses and the BVP describes the substrate concentration inside the biofilm. We show that this model can be reduced to a planar autonomous ODE that can be studied in the phase plane. Furthermore, we study the existence and uniqueness of solutions and characterize longterm behaviour. Lastly, we perform numerical simulations of the model to investigate the time evolution of the system, along with some sensitivity analysis to investigate the role of certain parameters.

\section{Mathematical model}

\subsection{Model assumptions}

1. We use the Wanner-Gujer 1-D biofilm model, i.e. we assume that the biofilm thickness is spatially constant, and the biomass density is also constant. Thus, the production of new biomass corresponds 1:1 to biofilm expansion [13]. Similarly, biomass loss corresponds 1:1 to biofilm contraction.

2. The biofilm is submerged in some aqueous solution of a substrate with a constant concentration. The substrate concentration inside the biofilm at the biofilm-aqueous solution layer is equal to the concentration of the aqueous solution.

3. The biofilm can degrade the substratum, which leads to a decrease in the thickness of the substratum. This degradation promotes bacterial growth inside the biofilm, which by assumption 1 implies an expansion of the biofilm. Furthermore, the biofilm degrades the substratum based on the substrate concentration at the biofilmsubstratum interface. If the concentration is too low, then no degradation will occur. If the threshold is exceeded, the degradation rate increases with the increase in concentration up to a constant rate.

\subsection{Governing equations}

\subsubsection{Substrate concentration in the biofilm and substrate flux between the aqueous phase and the biofilm}

The dissolved growth-limiting substrate inside the biofilm is transported via Fickian diffusion in the z-direction, the direction of biofilm growth, and is consumed by bacteria to promote growth. We apply the standard time scale argument in biofilm modelling that substrate diffusion and utilization are much quicker than biofilm growth. This allows for a quasi-steady-state assumption [13]. Let $\lambda$ be the thickness of the biofilm, then the 
substrate concentration $C(z)$ inside the biofilm is given by the solution to the two-point boundary value problem

$$
D_{c} \frac{d^{2} C}{d z^{2}}=\frac{\mu X}{Y} \frac{C}{K_{S}+C}, \quad \frac{d C}{d z}(0)=0, \quad C(\lambda)=C_{\infty}, \quad 0<z<\lambda,
$$

where $D_{c}$ is the diffusion coefficient, $X$ is the biomass density of the biofilm, $\mu$ is the per capita growth rate for the biofilm, $C_{\infty}$ is the concentration of substrate in the aqueous phase, also referred to as the bulk substrate concentration, $\mathrm{Y}$ is the biofilm yield coefficient, and $K_{S}$ is the biofilm half-saturation constant. $\mu C /\left(K_{S}+C\right)$ is the Monod kinetics, which is the standard uptake function used in biofilm modelling $[2,5,9,13]$. The location $z=0$ is the biofilm/substratum interface layer and $z=\lambda$ is the biofilm/bulk aqueous phase layer. The Fickian flux $J$ of substrate into the biofilm, which describes the transport of substrate into and throughout the biofilm, is given by

$$
J\left(\lambda ; C_{\infty}\right)=\frac{Y D_{c}}{X} \frac{d C}{d z}(\lambda)
$$

\subsubsection{Biofilm and substratum thickness model}

We determine the biofilm thickness $\lambda$ from a mass balance of biofilm growth, detachment, and substratum degradation. In our model formulation, we use a generic detachment function $d$ and a generic substratum degradation rate function $g$, both of which are made more precise later. Therefore, we can calculate the change in biofilm thickness as

$$
\frac{d \lambda}{d t}=\int_{0}^{\lambda} \frac{\mu C(z)}{K_{S}+C(z)} d z-\int_{0}^{\lambda} k_{l} d z-\int_{0}^{\lambda} d(\lambda) d z+A g(C(0)) H(S) L(\lambda),
$$

where $k_{l}$ is the natural cell lysis rate, $\mathrm{A}$ is the yield coefficient between substratum degradation and biofilm growth, $g$ is the rate of substratum degradation, which is a function of $C(0)$ by model assumption $3, d$ is the detachment rate function, and $S$ is the thickness of the substratum. The three integrals in (3) correspond to the total growth/loss from substrate consumption, cell lysis, and detachment, respectively. We assume that the coordinates placed on our system always put $z=0$ at the biofilm-substratum interface, which gives the limits of integration 0 to $\lambda$ as is used above. Lastly,

$$
L(\lambda):=\frac{\lambda}{\lambda+\epsilon_{\lambda}} \text { and } H(S):=\frac{S}{S+\epsilon_{S}}
$$

are approximations of the Heaviside function. We introduce these terms since no degradation should occur when $\lambda=0$ or $S=0$, but the degradation rate should be approximately $g$ whenever $S$ and $\lambda$ are positive.

The substratum cannot grow and is solely degraded by the biofilm, so we write the change in substratum thickness as

$$
\frac{d S}{d t}=-g(C(0)) H(S) L(\lambda)
$$

If we integrate (1) from 0 to $\lambda$ and apply the no flux boundary condition we obtain

$$
\int_{0}^{\lambda} \frac{\mu C}{K_{S}+C} d z=\frac{Y D_{c}}{X} \frac{d C}{d z}(\lambda)=J\left(\lambda ; C_{\infty}\right)
$$


We note that the concentration at the biofilm-substratum interface $C(0)$, is solely a function of $\lambda$ by (1). Therefore, we define $G(\lambda):=g(C(0))$; this allows us to write our model for biofilm and substratum thickness as an autonomous system of ODEs given by

$$
\begin{aligned}
& \frac{d \lambda}{d t}=J\left(\lambda ; C_{\infty}\right)-\left(k_{l}+d(\lambda)\right) \lambda+A G(\lambda) H(S) L(\lambda), \\
& \frac{d S}{d t}=-G(\lambda) H(S) L(\lambda) .
\end{aligned}
$$

Furthermore, we assume that $g$ and $d$ are sufficiently smooth and that $g$ satisfies $g(C)=0$ for all $C \in[0, \tilde{c}], d g / d C>0$ for all $C>\tilde{c}$, and $\lim _{C \rightarrow \infty} g(C)=k$ where $\tilde{c}$ is the threshold concentration described in model assumption 3. These conditions on $g$ all follow from model assumption 3. We also assume that the function $d$, along with both its first and second derivatives, are all non-negative.

Remark 2.1. The conditions on the detachment rate function d satisfy some of the standard detachment rate functions used in the literature. These include,

- $d(\lambda)=$ const

- $d(\lambda)=\delta \lambda^{\gamma}, \gamma \geq 1, \delta \geq 0$

as well as certain shear dependent detachment functions described in [1].

Lastly, for the purposes of this work, we assume that all parameters are positive.

\section{Properties of the mathematical model}

In this section we outline important properties related to (1) and (5). We begin by showing that any solution to (1) is non-negative and less than or equal to the bulk substrate concentration.

Proposition 3.1. Any solution, $C=C(z)$, to (1) satisfies $0 \leq C(z) \leq C_{\infty}$.

Proof. We begin by showing that $C(z)$ is non-negative. To do this we consider the differential equation

$$
\frac{d^{2} c}{d x^{2}}=k_{1} \frac{|c|}{k_{2}+|c|}, \quad 0<x<1
$$

where $k_{1}, k_{2}>0$. We consider (6) with boundary conditions

$$
\frac{d c}{d x}(0)=0 \text { and } c(1)=1 .
$$

Since $|c| \geq 0$ we have

$$
k_{1} \frac{|c|}{k_{2}+|c|} \leq \frac{k_{1}}{k_{2}}|c| .
$$

Let $c_{1}(x)$ denote the solution to

$$
\frac{d^{2} c}{d x^{2}}=\frac{k_{1}}{k_{2}}|c|, \quad 0<x<1
$$


with the boundary conditions (7). Then by comparison theorems for Sturm-Liouville type boundary value problems [12], we get $c_{1}(x) \leq c(x)$. Solving (8) with (7) gives

$$
c_{1}(x)=\operatorname{sech}\left(\sqrt{\frac{k_{1}}{k_{2}}}\right) \cosh \left(\sqrt{\frac{k_{1}}{k_{2}}} x\right)
$$

Since $c_{1}(x)>0$ we have that $c(x) \geq 0$. Therefore, this solution satisfies

$$
\frac{d^{2} c}{d x^{2}}=k_{1} \frac{c}{k_{2}+c}, \quad \frac{d c}{d x}(0)=0, \quad c(1)=1 .
$$

with (7). Since (10) is the non-dimensionalized version of (1) with $x=z / \lambda, c=C / C_{\infty}$, $k_{1}=\mu X \lambda^{2} /\left(C_{\infty} Y D_{c}\right)$, and $k_{2}=K_{S} / C_{\infty}$, we have $C(z) \geq 0$ where $C(z)$ is a solution to (1). Now because $C(z) \geq 0$ we know that

$$
\frac{d^{2} C}{d z^{2}}=\frac{\mu X}{Y D_{c}} \frac{C}{K_{S}+C} \geq 0 .
$$

Therefore, we know the maximum value of $C$ must occur on the boundary. Since

$$
\frac{d C}{d z}(0)=0 \text { and } \frac{d^{2} C}{d z^{2}} \geq 0
$$

we have that $C(0) \leq C(\lambda)$. Therefore, $C(z) \leq C(\lambda)=C_{\infty}$.

Remark 3.2. Since $0 \leq C(z) \leq C_{\infty}$, we can define upper and lower estimates on $c(x)$ using comparison theorems for Sturm-Liouville type boundary value problems [12]. Since

$$
\frac{k_{1}}{k_{2}+1} c \leq \frac{k_{1} c}{k_{2}+c} \leq \frac{k_{1}}{k_{2}} c
$$

we have

$$
\operatorname{sech}\left(\sqrt{\frac{k_{1}}{k_{2}}}\right) \cosh \left(\sqrt{\frac{k_{1}}{k_{2}}} x\right) \leq c(x) \leq \operatorname{sech}\left(\sqrt{\frac{k_{1}}{k_{2}+1}}\right) \cosh \left(\sqrt{\frac{k_{1}}{k_{2}+1}} x\right) .
$$

Reversing the non-dimensionalization and noting

$$
\frac{k_{1}}{k_{2}+1}=\frac{\theta \lambda^{2}}{K_{S}+C_{\infty}} \text { and } \frac{k_{1}}{k_{2}}=\frac{\theta \lambda^{2}}{K_{S}}
$$

where $\theta:=\mu X /\left(Y D_{c}\right)$, we obtain

$$
\operatorname{sech}\left(\sqrt{\frac{\theta \lambda^{2}}{K_{S}}}\right) \cosh \left(\sqrt{\frac{\theta}{K_{S}}} z\right) \leq \frac{C(z)}{C_{\infty}} \leq \operatorname{sech}\left(\sqrt{\frac{\theta \lambda^{2}}{K_{S}+C_{\infty}}}\right) \cosh \left(\sqrt{\frac{\theta}{K_{S}+C_{\infty}} z}\right) .
$$

We define

$$
\begin{aligned}
& c_{1}(x):=\operatorname{sech}\left(\sqrt{\frac{k_{1}}{k_{2}}}\right) \cosh \left(\sqrt{\left.\frac{k_{1}}{k_{2}} x\right),}\right. \\
& c_{2}(x):=\operatorname{sech}\left(\sqrt{\frac{k_{1}}{k_{2}+1}}\right) \cosh \left(\sqrt{\frac{k_{1}}{k_{2}+1}} x\right), \\
& C_{1}(z):=C_{\infty} \operatorname{sech}\left(\sqrt{\frac{\theta \lambda^{2}}{K_{S}}}\right) \cosh \left(\sqrt{\frac{\theta}{K_{S}}} z\right), \\
& C_{2}(z):=C_{\infty} \operatorname{sech}\left(\sqrt{\frac{\theta \lambda^{2}}{K_{S}+C_{\infty}}}\right) \cosh \left(\sqrt{\frac{\theta}{K_{S}+C_{\infty}}} z\right) .
\end{aligned}
$$


Now we show that a unique solution to (1) exists.

Proposition 3.3. There exists a unique solution to (1).

Proof. We first show the existence of a solution by considering the two point boundary value problem

$$
\frac{d^{2} C}{d z^{2}}=\frac{\mu X}{Y} \frac{|C|}{K_{S}+|C|}, \quad \frac{d C}{d z}(0)=0, \quad C(\lambda)=C_{\infty}, \quad 0<z<\lambda .
$$

From [12] we have that a solution to (11) exists if

$$
f(C)=\frac{\mu X}{Y} \frac{|C|}{K_{S}+|C|}
$$

is continuous and bounded, and the corresponding homogeneous problem

$$
\frac{d^{2} C}{d z^{2}}=0, \quad \frac{d C}{d z}(0)=0, \quad C(\lambda)=0, \quad 0<z<\lambda,
$$

has only the trivial solution. We note that $f(C)$ is continuous and bounded, since $K_{S}>0$ and $0 \leq f(C) \leq \mu X / Y$. From [12] we have that (12) possess only the trivial solution if and only if

$$
\operatorname{det}\left(\begin{array}{ll}
\frac{d C_{a}}{d z}(0) & \frac{d C_{b}}{d z}(0) \\
C_{a}(\lambda) & C_{b}(\lambda)
\end{array}\right) \neq 0
$$

where $C_{a}$ and $C_{b}$ form any fundamental solution set to $d^{2} C / d z^{2}=0$. A fundamental set of solutions to (12) is $C_{a}(z)=z$ and $C_{b}(z)=1$. Substituting into (13) gives

$$
\operatorname{det}\left(\begin{array}{ll}
1 & 0 \\
\lambda & 1
\end{array}\right)=1 \neq 0
$$

From Proposition 3.1 we know that solutions to (11) are non-negative. Therefore, any solution to (11) is a solution to (1).

To show uniqueness we consider the two point boundary value problem

$$
\frac{d^{2} c}{d x^{2}}=f(c):=k_{1} \frac{c}{k_{2}+c}, \quad-1<x<1, \quad c(-1)=1=c(1) .
$$

where $k_{1}, k_{2}>0$. Let $c_{1}$ and $c_{2}$ be two solutions to (14) and let $w:=c_{1}-c_{2}$. Since $c_{1}$ and $c_{2}$ are continuous functions we know that either $w \equiv 0$ or there are open intervals for which $c_{1}>c_{2}$ or $c_{1}<c_{2}$. Since $f$ is monotonically increasing we know that if $w>0$, then $d^{2} w / d x^{2}>0$ and if $w<0$, then $d^{2} w / d x^{2}<0$. Let $(a, b)$ be an interval where $w>0$ and $w(a)=0=w(b)$. Then by the mean value theorem, there must exist a maximum in $(a, b)$, but since $w$ is twice differentiable we know that $d^{2} w / d x^{2}<0$ at the maximum. Therefore, there exists no intervals for which $w>0$. Similarly, if $w<0$ on an interval $(c, d)$ and $w(c)=0=w(d)$, then $w$ must attain a minimum but $d^{2} w / d x^{2}<0$. Therefore, there are no intervals where $w<0$ and hence $c_{1}-c_{2}=w \equiv 0$.

Now let $c=\tilde{c}(x)$ be a solution to (10). Then $c=\tilde{c}(-x)$ is a solution to

$$
\frac{d^{2} c}{d x^{2}}=k_{1} \frac{c}{k_{2}+c}, \quad-1<x<0, \quad \frac{d c}{d x}(0)=0, \quad c(-1)=1 .
$$


Therefore,

$$
c(x):=\left\{\begin{array}{ll}
\tilde{c}(x) & 0 \leq x \leq 1 \\
\tilde{c}(-x) & -1 \leq x<0
\end{array} .\right.
$$

is a solution to (14). Since there exists exactly one solution to (14), we know that the solution to (10) is unique. Since (10) is a non-dimensionalized version of (1), see Proposition 3.1 and Remark 3.2, we conclude that solutions to (1) are unique.

Proposition 3.4. $G(\lambda):=g(C(0))$ is a decreasing function.

Proof. We again consider the non-dimensionalization of (1), i.e. (10). We see that if $\lambda_{1}>\lambda_{2}>0$, then $d^{2} c_{1} / d x^{2}>d^{2} c_{2} / d x^{2}$ where $d^{2} c_{1} / d x^{2}$ and $d^{2} c_{2} / d x^{2}$ denote the left hand side of (10) with $\lambda=\lambda_{1}$ and $\lambda=\lambda_{2}$ plugged in respectively. Then denoting $c_{1}(x)$ and $c_{2}(x)$ as the respective solutions we get that $c_{1}(x)<c_{2}(x)$, by comparison theorems for Sturm-Liouville type boundary value problems [12]. Thus taking $x=0$ we have $c_{1}(0)<c_{2}(0)$. Reversing the non-dimensionalization gives $C_{1}(0)<C_{2}(0)$. Since $g(C)$ is increasing we see that $G(\lambda)$ is decreasing.

With these results established we move on to properties of the flux function. The results given below were all proved in [9], with the exception of property (4), which was shown in [7]. We give a quick sketch of the proof.

Theorem 3.5 (Properties of $J\left(\lambda ; C_{\infty}\right)$ ). The flux function $J\left(\lambda ; C_{\infty}\right)$ for $\lambda \geq 0, C_{\infty} \geq 0$ is well defined, non-negative, differentiable, and monotonically increasing. Furthermore $J\left(\lambda ; C_{\infty}\right)$ has the following properties:

(1) $J(\lambda ; 0)=0=J\left(0 ; C_{\infty}\right)$.

(2) $\frac{\mu C_{\infty}}{K_{S}+C_{\infty}} \leq \frac{\partial J}{\partial \lambda}\left(0 ; C_{\infty}\right) \leq \frac{\mu C_{\infty}}{K_{S}}$.

(3) $C_{\infty} \sqrt{\frac{\phi}{K_{S}+C_{\infty}}} \tanh \left(\sqrt{\frac{\theta}{K_{S}+C_{\infty}}} \lambda\right) \leq J\left(\lambda ; C_{\infty}\right) \leq C_{\infty} \sqrt{\frac{\phi}{K_{S}}} \tanh \left(\sqrt{\frac{\theta}{K_{S}}} \lambda\right)$ where $\phi:=\mu Y D_{c} / X$.

(4) $\frac{\partial^{2} J}{\partial \lambda^{2}} \leq 0$

Here we give a quick sketch of the proof of everything but property (4). By definition, the function $J\left(\lambda ; C_{\infty}\right)$ for $\lambda \geq 0$ and $C_{\infty} \geq 0$ is non-negative and monotonically increasing. Since $J\left(\lambda ; C_{\infty}\right)$ is defined by the derivative of the concentration gradient $C(z)$ we know that the function is well-defined and differentiable. Property (1) follows directly from the definition. Properties (2) and (3) are derived from the upper and lower solutions outlined in Remark 3.2.

\section{Definition 3.6.}

$$
\begin{aligned}
& J_{l}\left(\lambda, C_{\infty}\right):=C_{\infty} \sqrt{\frac{\phi}{K_{S}+C_{\infty}}} \tanh \left(\sqrt{\frac{\theta}{K_{S}+C_{\infty}}} \lambda\right), \\
& J_{u}\left(\lambda ; C_{\infty}\right):=C_{\infty} \sqrt{\frac{\phi}{K_{S}}} \tanh \left(\sqrt{\frac{\theta}{K_{S}}} \lambda\right) .
\end{aligned}
$$


The following result shows us that the model (5) is well-posed.

Theorem 3.7. The model (5) with non-negative initial data possesses a unique, nonnegative solution that exists for all $t>0$.

Proof. From Theorem 3.5 we know that $J\left(0 ; C_{\infty}\right)=0$. Therefore, using the tangent criterion from [12], it follows that the non-negative cone is positively invariant. Note that $C$ is differentiable with respect to $\lambda[12]$, as $\lambda$ is a parameter, so $G(\lambda)$ is continuously differentiable by the properties of $g$. Hence, in the non-negative cone, the right hand sides of (5) are continuously differentiable, so the system satisfies a Lipschitz condition. This implies the local existence and uniqueness of a non-negative solution of the initial value problem (5) with non-negative initial data. Since $d S / d t \leq 0$, we have that $0 \leq S \leq S(0)$. Furthermore, there exists a positive constant $K_{1}$ such that $K_{1}>A G(\lambda) H(S) L(\lambda)$. Since $J_{u}\left(\lambda ; C_{\infty}\right)$ is bounded, we know that $J\left(\lambda ; C_{\infty}\right)<K_{2}$ for some positive constant $K_{2}$. Therefore, the solution, $y=y(t)$, to $d y / d t=K_{1}+K_{2}-k_{l} y$ is an upper bound for $\lambda(t)$. Since $y(t)$ is bounded, we know that $\lambda(t)$ is bounded above. Therefore, (5) possesses a unique, non-negative solution that exists for all $t>0$.

\section{Equilibrium and stability analysis}

Proposition 4.1. A line of trivial equilibria, $(\lambda, S)=(0, S)$, of the system defined by (5), exists for all parameters.

Proof. By Proposition 3.5 property $(1), J\left(0 ; C_{\infty}\right)=0$. Furthermore, $L(0)=0$. Thus, if $\lambda=0$, then $d S / d t=0$ and $d \lambda / d t=0$.

Since equilibrium values of $(5)$ must have $0=G(\lambda) H(S) L(\lambda)$ for every $S$, we perform analysis of the biofilm model without substratum degradation, i.e.

$$
\frac{d \lambda}{d t}=J\left(\lambda ; C_{\infty}\right)-\left(k_{l}+d(\lambda)\right) \lambda .
$$

Lemma 4.2. There are at most two non-negative solutions to

$$
J\left(\lambda ; C_{\infty}\right)-\left(k_{l}+d(\lambda)\right) \lambda=0 .
$$

The first is the trivial solution $\lambda=0$, which exists for all parameters and the second is a positive solution, which depends on model parameters and exists if and only if

$$
\frac{\partial J}{\partial \lambda}\left(0 ; C_{\infty}\right)>k_{l}+d(0)
$$

Proof. The existence of the trivial equilibrium follows directly from Theorem 3.5 property (1). For the second solution we consider the two functions

$$
\begin{aligned}
& f(\lambda):=J\left(\lambda ; C_{\infty}\right) \\
& h(\lambda):=\left(k_{l}+d(\lambda)\right) \lambda
\end{aligned}
$$

where we note that the intersection points of $f$ and $h$ are the only solutions to (18). We have that $h(0)=0$ and from the properties of $d(\lambda)$ we have that $d h / d \lambda>0$ and $d^{2} h / d \lambda^{2} \geq 0$ for all $\lambda \geq 0$. From Theorem 3.5 we have that $f(0)=0, d f / d \lambda \geq 0$ and $d^{2} f / d \lambda^{2} \leq 0$. Therefore, the non-trivial solution will exist if and only if $d f / d \lambda(0)>$ $d h / d \lambda(0)$ and is unique, since $d^{2} f / d \lambda^{2} \leq 0$ and $d^{2} h / d \lambda^{2} \geq 0$. 


\section{Remark 4.3.}

- From the proof given above we see that if $\lambda^{*}>0$ is the non-trivial solution to (18), then $J\left(\lambda ; C_{\infty}\right)>\left(k_{l}+d(\lambda)\right) \lambda$ for all $\lambda<\lambda^{*}$ and $J\left(\lambda ; C_{\infty}\right)<\left(k_{l}+d(\lambda)\right) \lambda$ for all $\lambda>\lambda^{*}$. Furthermore, if $\lambda^{*}$ does not exist, then $J\left(\lambda ; C_{\infty}\right)-\left(k_{l}+d(\lambda)\right) \lambda<0$.

- The functions $J_{u}$ and $J_{l}$, which denote the upper and lower solutions of the flux function $J$ respectively, can both be written in the form $a \tanh (b \lambda)$ where $a$ and $b$ are positive constants. Therefore, we know that both estimates satisfy the criteria used in the above proof. Therefore, each $J_{i}\left(\lambda ; C_{\infty}\right)=\left(k_{l}+d(\lambda)\right) \lambda$, for $i \in\{l, u\}$ has at most two solutions where the non-trivial solution exists if and only if

$$
\frac{\partial J_{i}}{\partial \lambda}(0)>k_{l}+d(0)
$$

We will use these results to give sufficient conditions for the existence and uniqueness of non-trivial equilibria of (5).

The function $J\left(\lambda, C_{\infty}\right)$ and its derivatives are not easy to evaluate. However, using the estimates from Theorem 3.5 we can derive the following weaker criterion for the existence of the non-trivial solution to (17).

Corollary 4.4. If $\mu C_{\infty} /\left(K_{S}+C_{\infty}\right)>k_{l}+d(0)$, then the non-trivial solution to (18) exists.

Proof. This follows directly from Theorem 3.5 property (2) and Lemma 4.2.

We now give sufficient conditions for which $G$ vanishes and for which $G$ is positive.

Proposition 4.5. Let

$$
\eta:=\sqrt{\frac{Y D_{c}\left(K_{S}+C_{\infty}\right)}{\mu X}} .
$$

If $\tilde{c}<C_{\infty}$ and $\lambda \geq \eta \operatorname{arcsech}\left(\tilde{c} / C_{\infty}\right)$, then $G(\lambda)=0$. Furthermore, if $\tilde{c} \geq C_{\infty}$, then $G(\lambda)=0$.

Proof. Suppose $\tilde{c} \geq C_{\infty}$. Then by Proposition 3.1, $C(0) \leq \tilde{c}$. Therefore, $G(\lambda)=$ $g(C(0))=0$. Now suppose $\tilde{c}<C_{\infty}$. Then, $G(\lambda)=g(C(0))=0$ whenever $C(0) \in[0, \tilde{c}]$. Using the upper estimate from Remark 3.2 we have that $C(0) \leq C_{2}(0)$. Therefore, if $C_{2}(0)=C_{\infty} \operatorname{sech}(\lambda / \eta) \leq \tilde{c}$, then $C(0) \leq \tilde{c}$. Thus $G(\lambda)=0$. Suppose

$$
\lambda \geq \eta \operatorname{arcsech}\left(\frac{\tilde{c}}{C_{\infty}}\right),
$$

then since $0 \leq \tilde{c}<C_{\infty}$, the range of $\operatorname{arcsech}(x)$ is $[0, \infty)$, and $\operatorname{sech}(x)$ is a decreasing function for $[0, \infty)$ we can rearrange (19) to obtain

$$
C_{\infty} \operatorname{sech}\left(\frac{\lambda}{\eta}\right) \leq \tilde{c}
$$


Proposition 4.6. Let

$$
\eta_{0}:=\sqrt{\frac{Y D_{c} K_{S}}{\mu X}} .
$$

If $\tilde{c}<C_{\infty}$ and $\lambda<\eta_{0} \operatorname{arcsech}\left(\tilde{c} / C_{\infty}\right)$, then $G(\lambda)>0$.

Proof. Suppose $\tilde{c}<C_{\infty}$. Then, $G(\lambda)=g(C(0))>0$ whenever $C(0)>\tilde{c}$. Using the lower estimate from Remark 3.2 we have $C_{1}(0) \leq C(0)$. Therefore, if $\tilde{c}<C_{\infty} \operatorname{sech}\left(\lambda / \eta_{0}\right)=$ $C_{1}(0)$, then $\tilde{c}<C(0)$. Thus $G(\lambda)>0$. Suppose

$$
\lambda<\eta_{0} \operatorname{arcsech}\left(\frac{\tilde{c}}{C_{\infty}}\right),
$$

then since $0 \leq \tilde{c}<C_{\infty}$, the range of $\operatorname{arcsech}(x)$ is $[0, \infty)$, and $\operatorname{sech}(x)$ is a decreasing function for $[0, \infty)$ we can rearrange $(20)$ to obtain

$$
\tilde{c}<C_{\infty} \operatorname{sech}\left(\frac{\lambda}{\eta_{0}}\right) .
$$

From Lemma 4.2 and Proposition 4.5 we see that there are two critical $\lambda$ values in our model. These values correspond to the non-trivial solution to (18) and the threshold value for which $G(\lambda)=0$. We define these critical points below.

Definition 4.7. Let $\tilde{\lambda}$ be the critical $\lambda$ value for which $G(\lambda)=0$ for all $\lambda \geq \tilde{\lambda}$ and $G(\lambda)>0$ for all $\lambda<\tilde{\lambda}$. Furthermore, let $\lambda^{*}$ denote the unique positive solution to (18).

\section{Remark 4.8.}

- By Proposition 4.5, $\tilde{\lambda}$ always exists and is always non-negative. By Lemma 4.2 and Corollary $4.4, \lambda^{*}$ exists under certain conditions on the growth of the biofilm.

- If $\tilde{c} \geq C_{\infty}$, then $\tilde{\lambda}=0$. Furthermore, if $\tilde{c}<C_{\infty}$, then $\tilde{\lambda}>0$.

- $\lambda^{*}$ and $\tilde{\lambda}$ can be controlled independently. This leads to three different cases:
1. $\lambda^{*}$ exists and $\lambda^{*}>\tilde{\lambda}$,
2. $\lambda^{*}$ exists and $\lambda^{*}<\tilde{\lambda}$,
3. $\lambda^{*}$ does not exist.

We will show below that each case has its own distinct long-term behaviour. Furthermore, $\lambda^{*}$ and $\tilde{\lambda}$ cannot be computed easily, thus for each case we give a sufficient condition, which depends only on model parameters, that guarantees each case holds. 


\subsection{Case 1: $\lambda^{*}$ exists and $\lambda^{*}>\tilde{\lambda}$}

Theorem 4.9. If $\lambda^{*}$ exists and $\lambda^{*}>\tilde{\lambda}$, then there exists a line of non-trivial equilibria, $(\lambda, S)=\left(\lambda^{*}, S\right)$.

Proof. Suppose $\lambda^{*}>\tilde{\lambda} \geq 0$ are fixed. Then by the definition of $\tilde{\lambda}$, we have that $d S / d t=0$ at $\lambda^{*}$ and since $\lambda^{*}$ is the unique non-trivial solution for which (17) vanishes, we have $d \lambda / d t=0$ for every $S \geq 0$.

We now give a weaker condition for the existence of the line of non-trivial equilibria, which depends only on model parameters.

Corollary 4.10. If either of these two conditions holds

1. $\tilde{c} \geq C_{\infty}$ and $k_{l}+d(0)<\frac{\mu C_{\infty}}{K_{S}+C_{\infty}}$,

2. $\tilde{c}<C_{\infty}$ and

$$
k_{l}+d\left(\eta \operatorname{arcsech}\left(\frac{\tilde{c}}{C_{\infty}}\right)\right)<\frac{\mu C_{\infty} \sqrt{\left(1-\left(\tilde{c} / C_{\infty}\right)^{2}\right)}}{\left(K_{S}+C_{\infty}\right) \operatorname{arcsech}\left(\frac{\tilde{c}}{C_{\infty}}\right)}
$$

Then there exists a line of non-trivial equilibria, $(\lambda, S)=\left(\lambda^{*}, S\right)$.

Proof. We show that when either of these two conditions holds, $\lambda^{*}$ exists and $\lambda^{*}>\tilde{\lambda}$. Since $d(\lambda)$ is non-decreasing and $\left.0 \leq \sqrt{1-\left(\tilde{c} / C_{\infty}\right)^{2}} / \operatorname{arcsech}\left(\tilde{c} / C_{\infty}\right)\right) \leq 1$ we have

$$
k_{l}+d(0) \leq k_{l}+d\left(\eta \operatorname{arcsech}\left(\frac{\tilde{c}}{C_{\infty}}\right)\right)<\frac{\mu C_{\infty} \sqrt{\left(1-\left(\tilde{c} / C_{\infty}\right)^{2}\right)}}{\left(K_{S}+C_{\infty}\right) \operatorname{arcsech}\left(\frac{\tilde{c}}{C_{\infty}}\right)} \leq \frac{\mu C_{\infty}}{K_{S}+C_{\infty}} .
$$

We note that $k_{l}+d(0)<\mu C_{\infty} /\left(K_{S}+C_{\infty}\right)$, which is satisfied by both conditions, guarantees the existence of $\lambda^{*}$ from Corollary 4.4 and the non-trivial solution to $J_{l}\left(\lambda ; C_{\infty}\right)=\left(k_{l}+\right.$ $d(\lambda)) \lambda$. If $\tilde{c} \geq C_{\infty}$, then from Proposition 4.5 we have that $\tilde{\lambda}=0$. Therefore, $\lambda^{*}>\tilde{\lambda}$. Now suppose $\tilde{c}<C_{\infty}$ and let $\lambda=\lambda_{1}$ denote the non-trivial solution to $J_{l}\left(\lambda ; C_{\infty}\right)=\left(k_{l}+d(\lambda)\right) \lambda$. By Remark 4.3, we know that $\lambda_{1}$ is unique. Since $J_{l}\left(\lambda ; C_{\infty}\right) \leq J\left(\lambda, C_{\infty}\right)$ and $d(\lambda)$ is a non-decreasing function, we know $\lambda_{1} \leq \lambda^{*}$. Therefore, if $\lambda_{1}>\tilde{\lambda}$, then $\lambda^{*}>\tilde{\lambda}$. So if

$$
\lambda_{1}>\eta \operatorname{arcsech}\left(\frac{\tilde{c}}{C_{\infty}}\right),
$$

then by Proposition 4.5 and the definition of $\tilde{\lambda}, \lambda^{*}>\tilde{\lambda}$. We finish by showing that hypothesis (21) is sufficient for (22) to hold. By Remark 4.3 we know that if $k_{l}+d(\lambda)<$ $J_{l}\left(\lambda ; C_{\infty}\right) / \lambda$, then $\lambda<\lambda_{1}$. Since $\tanh (\operatorname{arcsech}(x))=\sqrt{1-x^{2}}$, we have that

$$
\frac{J_{l}\left(\eta \operatorname{arcsech}\left(\frac{\tilde{c}}{C_{\infty}}\right) ; C_{\infty}\right)}{\eta \operatorname{arcsech}\left(\frac{\tilde{c}}{C_{\infty}}\right)}=\frac{\mu \sqrt{\left(C_{\infty}^{2}-\tilde{c}^{2}\right)}}{\left(K_{S}+C_{\infty}\right) \operatorname{arcsech}\left(\frac{\tilde{c}}{C_{\infty}}\right)}
$$

Therefore, (21) guarantees that (22) holds.

Proposition 4.11. If $\lambda^{*}$ exists and $\lambda^{*}>\tilde{\lambda}$, then the only steady states of (5) are $(\lambda, S)=(0, S)$ and $(\lambda, S)=\left(\lambda^{*}, S\right)$. 
Proof. Suppose $\lambda^{*}$ exists and $\lambda^{*}>\tilde{\lambda}$. Then whenever $0<\lambda<\tilde{\lambda}$ we have by Remark 4.3 and the fact that $A G(\lambda) H(S) L(\lambda) \geq 0$ that $d \lambda / d t>0$. When $\lambda^{*} \neq \lambda$ and $\lambda>\tilde{\lambda}$ we have that $d \lambda / d t=J\left(\lambda ; C_{\infty}\right)-\left(k_{l}+d(\lambda)\right) \lambda \neq 0$ by Lemma 4.2 .

We now begin analysis of the long-term behaviour of the system whenever $\lambda^{*}$ exists and $\lambda^{*}>\tilde{\lambda}$. We first note that all equilibria points are non-hyperbolic. Therefore, we cannot use the traditional linearisation arguments to get a full picture of the long-term behaviour. We proceed by investigating solution trajectories in the phase plane.

Proposition 4.12. Suppose $\lambda^{*}$ exists and $\lambda^{*}>\tilde{\lambda}$. Then,

1. If $\lambda>\lambda^{*}$, then $d \lambda / d t<0$, and

2. If $0<\lambda<\lambda^{*}$, then $d \lambda / d t>0$.

Therefore, all non-constant solutions approach the line of non-trivial equilibria $\left(\lambda^{*}, S\right)$.

Proof. Follows directly from Lemma 4.2, Remark 4.3, and $A G(\lambda) H(S) L(\lambda) \geq 0$.

Figure 4.1 provides a reference phase portrait. As we can see the solution trajectories which start below $\lambda=\lambda^{*}$ travel upwards towards $\lambda=\lambda^{*}$. These trajectories also travel in the negative $S$-direction up until they reach $\lambda=\tilde{\lambda}$ where they then travel solely in the positive $\lambda$-direction. All solution trajectories which start above $\lambda=\lambda^{*}$ travel solely in the negative $\lambda$-direction towards $\lambda=\lambda^{*}$.

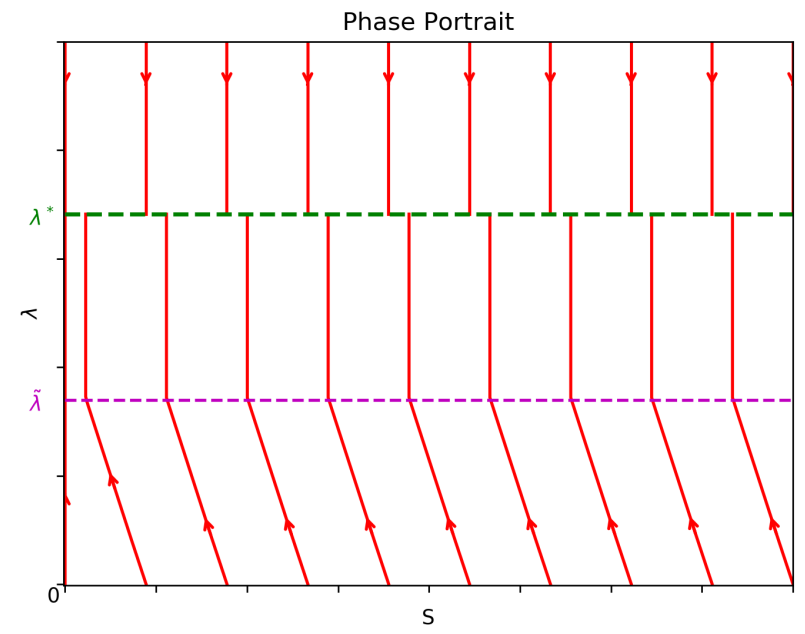

Figure 4.1: Sample phase portrait in the $S \lambda$-plane of (5), for long-term behaviour case 1. We see monotonic growth toward the final biofilm thickness. The trajectories at the bottom of the graph start at a small $\lambda$ value because the system (5) is in equilibrium whenever $\lambda=0$.

\subsection{Case 2: $\lambda^{*}$ exists and $\lambda^{*}<\tilde{\lambda}$}

Theorem 4.13. If $\lambda^{*}$ exists and $\lambda^{*}<\tilde{\lambda}$, then we have a non-trivial equilibrium point $\left(\lambda^{*}, 0\right)$. 
Proof. This follows directly from the definition of $\lambda^{*}$.

We now give a weaker criterion for which the equilibrium point $\left(\lambda^{*}, 0\right)$ exists.

Corollary 4.14. If $\tilde{c}<C_{\infty}, k_{l}+d(0)<\mu C_{\infty} /\left(K_{S}+C_{\infty}\right)$, and

$$
k_{l}+d\left(\eta_{0} \operatorname{arcsech}\left(\frac{\tilde{c}}{C_{\infty}}\right)\right)>\frac{\mu \sqrt{\left(C_{\infty}^{2}-\tilde{c}^{2}\right)}}{K_{S} \operatorname{arcsech}\left(\frac{\tilde{c}}{C_{\infty}}\right)}
$$

Then we have a non-trivial equilibrium point $\left(\lambda^{*}, 0\right)$.

Proof. We show that when the hypotheses hold, $\lambda^{*}$ exists and $\lambda^{*}<\tilde{\lambda}$. Suppose $\tilde{c}<C_{\infty}$. Since $k_{l}+d(0)<\mu C_{\infty} /\left(K_{S}+C_{\infty}\right)$ guarantees the existence of $\lambda^{*}$, and $J_{u} \geq J$ we have the existence of $\lambda=\lambda_{2}$ which is the solution to $J_{u}\left(\lambda ; C_{\infty}\right)=\left(k_{l}+d(\lambda)\right) \lambda$. By Remark 4.3, we know that $\lambda_{2}$ is unique. Since $J_{u}\left(\lambda ; C_{\infty}\right) \geq J\left(\lambda, C_{\infty}\right)$ and $d(\lambda)$ is a non-decreasing function, we know $\lambda_{2} \geq \lambda^{*}$. Therefore, if $\lambda_{2}<\tilde{\lambda}$, then $\lambda^{*}<\tilde{\lambda}$. So if

$$
\lambda_{2}<\eta_{0} \operatorname{arcsech}\left(\frac{\tilde{c}}{C_{\infty}}\right)
$$

then by Proposition 4.6 and the definition of $\tilde{\lambda}, \lambda^{*}<\tilde{\lambda}$. We finish by showing that hypothesis (23) is sufficient for (24) to hold. By Remark 4.3 we know that if $k_{l}+d(\lambda)>$ $J_{u}\left(\lambda ; C_{\infty}\right) / \lambda$, then $\lambda>\lambda_{2}$. Since $\tanh (\operatorname{arcsech}(x))=\sqrt{1-x^{2}}$, we have that

$$
\frac{J_{u}\left(\eta_{0} \operatorname{arcsech}\left(\frac{\tilde{c}}{C_{\infty}}\right) ; C_{\infty}\right)}{\eta_{0} \operatorname{arcsech}\left(\frac{\tilde{c}}{C_{\infty}}\right)}=\frac{\mu \sqrt{\left(C_{\infty}^{2}-\tilde{c}^{2}\right)}}{K_{S} \operatorname{arcsech}\left(\frac{\tilde{c}}{C_{\infty}}\right)} .
$$

Therefore, (23) guarantees that (24) holds.

Proposition 4.15. If $\lambda^{*}$ exists and $\lambda^{*}<\tilde{\lambda}$, then the only steady states of (5) are $(\lambda, S)=(0, S)$ and $\left(\lambda^{*}, 0\right)$.

Proof. Suppose $\lambda^{*}$ exists and $\lambda^{*}<\tilde{\lambda}$. Then by Lemma 4.2 we have that if $\tilde{\lambda}<\lambda$, then $d \lambda / d t \neq 0$. When $0<\lambda<\tilde{\lambda}$ and $\lambda \neq \lambda^{*}$, we have by the definition of $\tilde{\lambda}$ and $\lambda^{*}$ that either $d S / d t<0$ or $d \lambda / d t \neq 0$. When $\lambda=\lambda^{*}$ and $S>0$, we have $d S / d t<0$.

We now begin analysis of the long-term behaviour of (5) when $\lambda^{*}$ exists and $\lambda^{*}<\tilde{\lambda}$. We note again that not all equilibria points are hyperbolic. Therefore, we cannot use the traditional linearisation arguments to get a full picture of long-term behaviour. We proceed by investigating solution trajectories in the phase plane.

Proposition 4.16. Suppose $\lambda^{*}$ exists and $\lambda^{*}<\tilde{\lambda}$. Then

1. If $\lambda>\tilde{\lambda}$, then $d \lambda / d t<0$,

2. If $\lambda^{*}<\lambda<\tilde{\lambda}$ and $S>0$, then $d S / d t<0$,

3. If $0<\lambda<\lambda^{*}$ and $S>0$, then $d S / d t<0$ and $d \lambda / d t>0$,

4. If $\lambda>0$ and $S=0$, then $d S / d t=0$ and $\lambda \rightarrow \lambda^{*}$ as $t \rightarrow \infty$.

Therefore, all non-constant solutions approach $\left(\lambda^{*}, 0\right)$. 
Proof. We begin with property 1 . Suppose $\lambda>\tilde{\lambda}$, then by the definition of $\tilde{\lambda}$ and Proposition 4.5 we have that $d \lambda / d t=J\left(\lambda ; C_{\infty}\right)-\left(k_{l}+d(\lambda)\right) \lambda$. By Lemma 4.2 and Remark 4.3 we have that $d \lambda / d t<0$. Now we move on to property 2. Suppose $\lambda^{*}<\lambda<\tilde{\lambda}$ and $S>0$. Then $G(\lambda), H(S), L(\lambda)>0$, therefore $d S / d t<0$. For property 3 , we see that if $0<\lambda<\lambda^{*}<\tilde{\lambda}$ and $S>0$, then since $A G(\lambda) H(S) L(\lambda)>0$, we have $d \lambda / d t>0$ and $d S / d t<0$. We finish with property 4 . Suppose $\lambda>0$ and $S=0$. Then $d S / d t=0$, which implies $d \lambda / d t=J\left(\lambda ; C_{\infty}\right)-\left(k_{l}+d(\lambda)\right) \lambda$. From Lemma 4.2 and Remark 4.3 we know that if $\lambda<\lambda^{*}$, then $d \lambda / d t=J\left(\lambda ; C_{\infty}\right)-\left(k_{l}+d(\lambda)\right) \lambda>0$ and if $\lambda>\lambda^{*}$, then $d \lambda / d t=J\left(\lambda ; C_{\infty}\right)-\left(k_{l}+d(\lambda)\right) \lambda<0$. Therefore, $\lambda \rightarrow \lambda^{*}$ as $t \rightarrow \infty$.

Figure 4.2 provides a reference phase portrait. Solutions where $S, \lambda>0$ travel towards some $\lambda=\lambda^{\prime}$ value between $\lambda^{*}$ and $\tilde{\lambda}$. Solutions where $\lambda>\tilde{\lambda}$ travel solely in the negative $\lambda$-direction, while solutions where $\lambda<\tilde{\lambda}$ travel in the negative $S$-direction and towards $\lambda^{\prime}$. Once the solutions reach $\lambda^{\prime}$ they travel in the negative $S$-direction until $S=0$, then all trajectories travel solely in the $\lambda$-direction until they reach steady state at $(S, \lambda)=\left(0, \lambda^{*}\right)$. We see that the biofilm doesn't necessarily grow monotonically in this case.

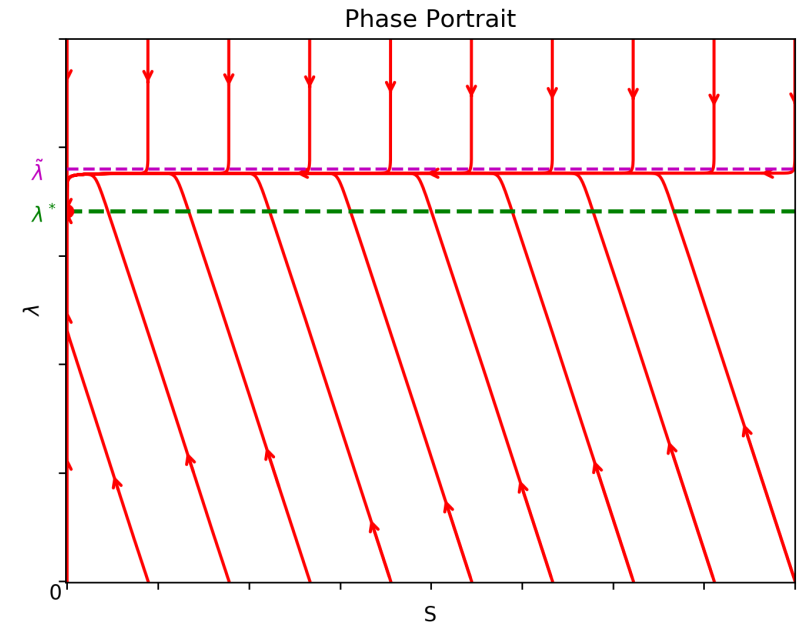

Figure 4.2: Sample phase portrait in the $S \lambda$-plane of (5), for long-term behaviour case 2. We see in this case that the biofilm thickness over shoots the final biofilm thickness $\lambda^{*}$ whenever $\lambda(0)<\lambda^{*}$. The trajectories at the bottom of the graph start at a small $\lambda$ value because the system (5) is in equilibrium whenever $\lambda=0$.

\subsection{Case 3: $\lambda^{*}$ does not exist}

Proposition 4.17. If $\lambda^{*}$ does not exist, then the line of trivial equilibria are the only equilibria of (5).

Proof. Suppose $\lambda^{*}$ does not exist. For any equilibrium to exist we need $G(\lambda) H(S) L(\lambda)=$ 0 . Therefore, $d \lambda / d t=J\left(\lambda ; C_{\infty}\right)-\left(k_{l}+d(\lambda)\right) \lambda$; which from Lemma 4.2 we know only vanishes if $\lambda=0$ or $\lambda=\lambda^{*}$. Since $\lambda^{*}$ does not exist, we conclude that $\lambda=0$ must hold for all equilibria of (5).

Corollary 4.18. If $\mu C_{\infty} / K_{S}<k_{l}+d(0)$, then the line of trivial equilibria are the only equilibria of (5). 
Proof. From Theorem 3.5 property (2) and Lemma 4.2 it follows that if $\mu C_{\infty} / K_{S}<$ $k_{l}+d(0)$, then $\lambda^{*}$ does not exist. So by Proposition 4.17, the line of trivial equilibria is the only equilibria of (5).

As all equilibrium points are non-hyperbolic, we move to analysis in the phase plane.

Proposition 4.19. Suppose $\lambda^{*}$ does not exist. Then $\lambda \rightarrow 0$ as $t \rightarrow \infty$.

Proof. Suppose $\lambda^{*}$ does not exist. Then if $0<\lambda<\tilde{\lambda}$ and $S>0$, then $d S / d t<0$. If $\lambda>\tilde{\lambda}$, then $d \lambda / d t<0$. Finally, if $\lambda>0$, and $S=0$, then by Remark $4.3, \lambda \rightarrow 0$.

Figure 4.3 provides a reference phase portrait. For $S>0$, solution trajectories travel towards a positive $\lambda=\lambda^{\prime}$ value less than $\tilde{\lambda}$. However, once $S=0$ the model simplifies to (17), which has trajectories decaying to $\lambda=0$ as $\lambda^{*}$ does not exist.

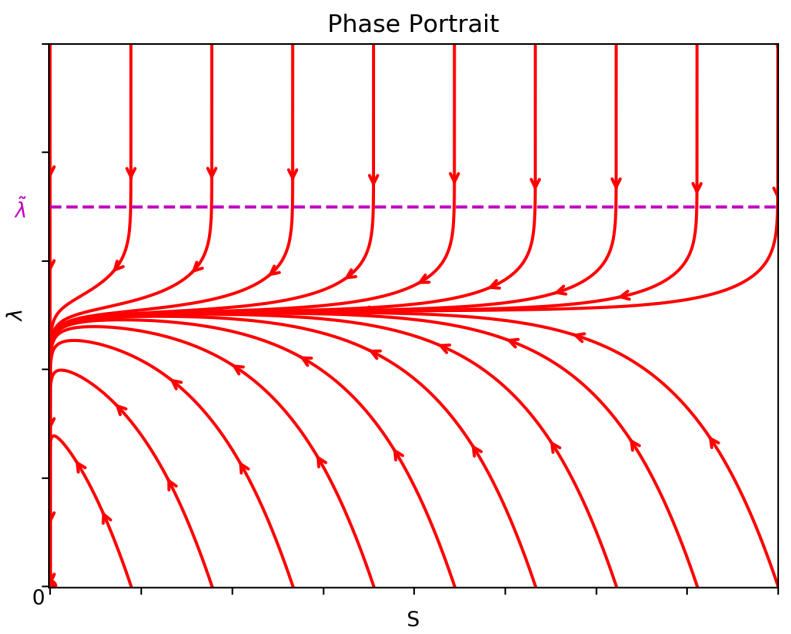

Figure 4.3: Sample phase portrait in the $S \lambda$-plane of (5), for long-term behaviour case 3. We see that the biofilm initially grows toward a positive thickness and then once $S=0$, the biofilm degrades down towards $\lambda=0$. The trajectories at the bottom of the graph start at a small $\lambda$ value because the system (5) is in equilibrium whenever $\lambda=0$.

In all cases of long-term behaviour the equilibria and the stability conditions of (5) are the same as in (17). Therefore, the long-term behaviour of (5) and (17) are the same.

\section{Simulations}

Numerical simulations of (5) were performed in Python using solve_ivp from the SciPy package [11]. This routine implements the Dormand-Prince-Runge-Kutta 4(5) method, see [4] for more details. The embedded two-point boundary value problem (1) was solved with the routine solve_bvp from the SciPy package [11]. This routine implements a 4th order collocation algorithm with the control of residuals similar to [6] and the collocation system is solved by a damped Newton method with an affine-invariant criterion function [3]. For all simulations, the model was solved until the euclidean norm of the right-hand side of (5) was less than $10^{-7}$, i.e a steady state was reached.

In the following sections, we provide simulation results of the system (5). Parameter values are summarized in Table 1, along with the definitions of $d$ and $g$ given by (25). 


\subsection{Simulation parameters}

For purposes of the simulation we assume the functions $d$ and $g$ are of the form

$$
d(\lambda)=\delta=\text { const }, \quad g(C)=\left\{\begin{array}{ll}
0 & 0 \leq C \leq \tilde{c} \\
k \frac{(C-\tilde{c})^{2}}{\kappa^{2}+(C-\tilde{c})^{2}} & C>\tilde{c}
\end{array} .\right.
$$

The function $d$ represents a constant detachment rate. The function $g$ is a Hill function, where for small values of $C(0), g(C(0))=0$ and for large values $g(C(0)) \approx k$. Since we rewrite $g(C(0))$ as $G(\lambda)$ in (5), we give two plots of the function $G(\lambda)=g(C(0)$ ), as described in (25), for $\lambda \in[0,0.0015], \tilde{c}=0.0002,0.002$, and the relevant parameters in Table 1 . The two values for $\tilde{c}$ are the values used in the simulations used to investigate the role of the biofilm/substratum yield coefficient $A$ and represent case 1 and case 2 of long-term behaviour under the parameters in Table 1. The plots are given in Figure 5.1.
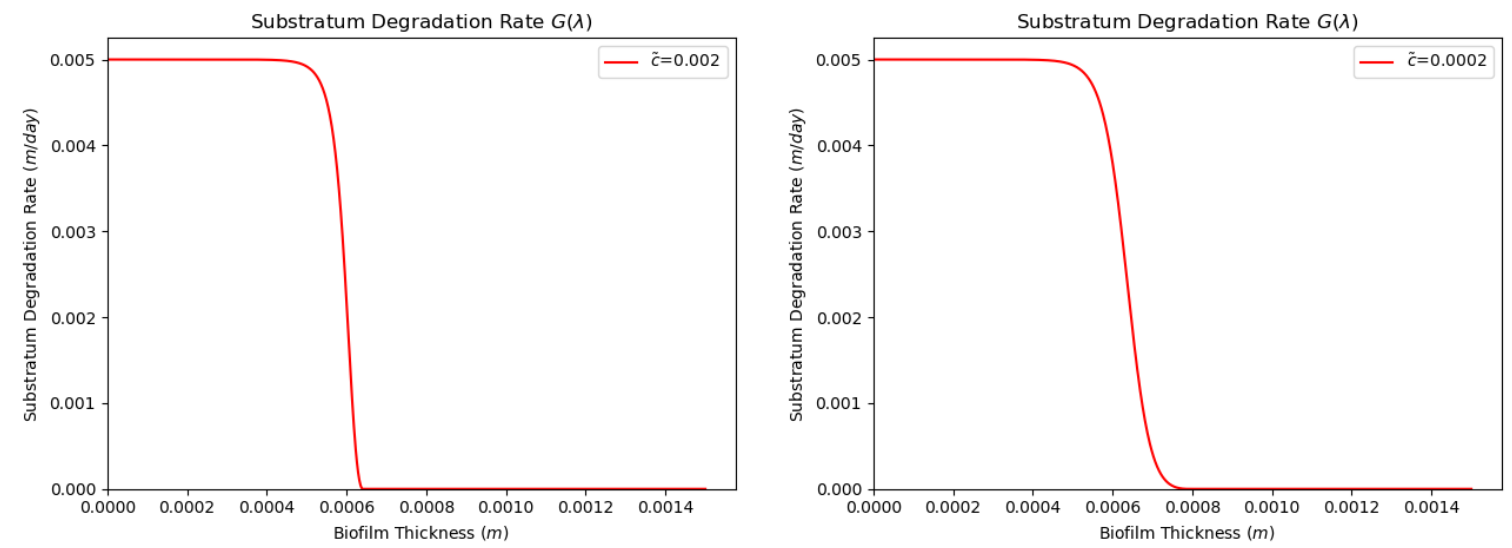

Figure 5.1: Plot of the substratum degradation rate function $G(\lambda)=g(C(0))$ for $g$ given in (25), $\lambda \in[0,0.0015], \tilde{c}=0.002$ (left), $\tilde{c}=0.0002$ (right), and $k$ and $\kappa$ given in Table 1.

\subsection{Effects of the threshold concentration}

In the first simulation, we investigate how the threshold concentration value affects the growth of the biofilm and the degradation of substratum when we are in the first case of long-term behaviour. In these simulations, we set the biofilm/substratum yield coefficient to $A=0.5$ and investigate threshold concentration values in the range of 0.002 to 0.005 . Furthermore, we compare these simulations to the base case biofilm model (17). The simulation results are reported in Figure 5.2.

We see that as the threshold concentration increases, the biofilm grows more slowly, and the substratum equilibrates at a larger final thickness. We also see that as the threshold concentration increases, the time to equilibration for the substratum thickness decreases. This is caused by the relationship between the threshold concentration and the value $\tilde{\lambda}$, as defined in Definition 4.7. Since $\tilde{\lambda}$ is the threshold value for which $G(\lambda)$ vanishes, and by considering the plots in Figure 5.1, we see that as $\tilde{c}$ decreases, $\tilde{\lambda}$ must increase and hence the substratum will be degraded for longer. This explains why the biofilm grows more slowly with larger threshold concentrations. We see that these simulations follow the first case of long-term behaviour; see Figure 4.1, i.e. $\lambda^{*}$ exists and 
Table 1: Model parameters used in simulations.

\begin{tabular}{lcccc}
\hline Parameter & Symbol & Value & Units & Reference \\
\hline Diffusion coefficient & $D_{c}$ & $10^{-4}$ & $\mathrm{~m}^{2} /$ day & {$[13]$} \\
Biofilm biomass density & $X$ & 10000 & $g / \mathrm{m}^{3}$ & {$[13]$} \\
Biofilm yield coefficient & $Y$ & 0.63 & - & {$[13]$} \\
Biofilm maximum growth rate & $\mu$ & 6 & $1 /$ day & {$[13]$} \\
Biofilm half saturation constant & $K_{S}$ & 4 & $g / \mathrm{m}^{3}$ & {$[13]$} \\
Aqueous phase concentration & $C_{\infty}$ & 10 & $g / \mathrm{m}^{3}$ & Assumed \\
Cell death rate & $k_{l}$ & 0.4 & $1 / d a y$ & {$[13]$} \\
Detachment coefficient & $\delta$ & 0.5 & $1 /$ day & {$[1]$} \\
Substratum maximal decay rate & $k$ & 0.005 & $1 /$ day & Assumed \\
Substratum decay half saturation constant & $\kappa$ & 0.002 & $g / m^{3}$ & Assumed \\
Threshold concentration & $\tilde{c}$ & Varied & $g / m^{3}$ & Assumed \\
Biofilm/substratum yield coefficient & $A$ & Varied & - & Assumed \\
Biofilm regularization parameter & $\epsilon_{\lambda}$ & $10^{-6}$ & $m$ & Assumed \\
Substratum regularization parameter & $\epsilon_{S}$ & $10^{-6}$ & $m$ & Assumed \\
Initial biofilm thickness & $\lambda_{0}$ & 0.00055 & $m$ & Assumed \\
Initial substratum thickness & $S_{0}$ & 0.0005 & $m$ & Assumed \\
\hline
\end{tabular}
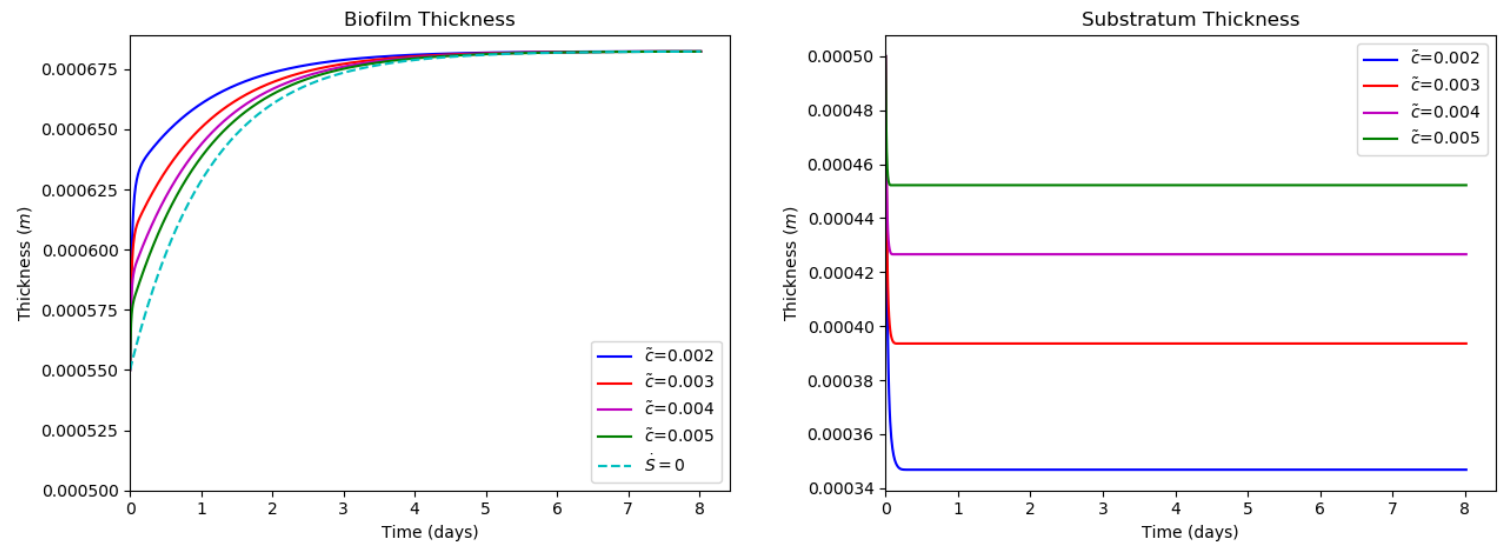

Figure 5.2: Comparison of the full system (5) and the base model (17) (dashed line), with varying threshold concentrations $\tilde{c}$. Each simulation uses the parameter values summarized in Table 1 with $\tilde{c}=\{0.002,0.003,0.004,0.005\}$ and $A=0.5$. The biofilm thickness $\lambda$ and substratum thickness $S$ are given on the left and right respectively. This simulation follows the first case of long-term behaviour.

$\lambda^{*}>\tilde{\lambda}$, since the substratum thicknesses are equilibrating at positive values. Lastly, as shown in the analysis, all final biofilm thicknesses are the same as in the base case model.

In the second simulation, we investigate how the threshold concentration value affects the growth of the biofilm and the degradation of the substratum when we are in the second case of long-term behaviour. In these simulations, we set the biofilm/substratum yield coefficient to $A=0.5$ and investigate threshold concentration values in the range of 0.0002 to 0.0005 . Moreover, we compare these simulations with the base case biofilm model (17). The simulation results are reported in Figure 5.3.

We first note that the biofilm thickness, in the full model, reaches a maximum thick- 

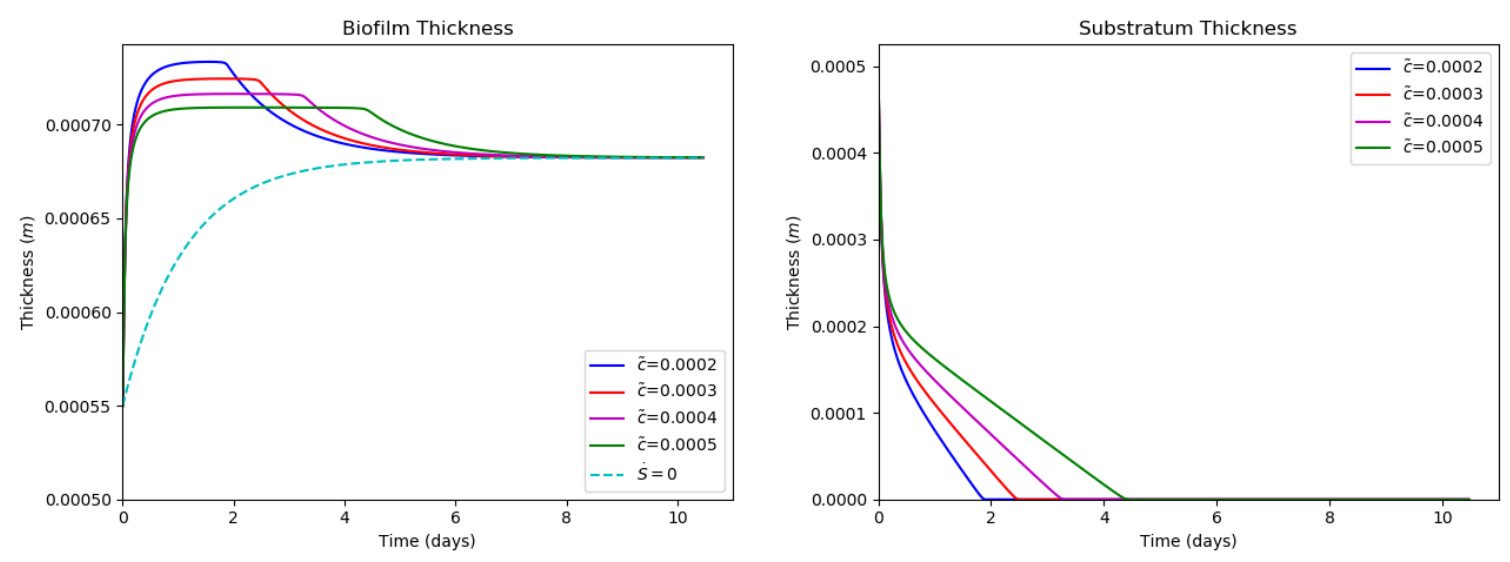

Figure 5.3: Comparison of the full system (5) and the base model (17) (dashed line), with varying threshold concentrations $\tilde{c}$. Each simulation uses the parameter values summarized in Table 1 with $\tilde{c}=\{0.0002,0.0003,0.0004,0.0005\}$ and $A=0.5$. The biofilm thickness $\lambda$ and substratum thickness $S$ are given on the left and right respectively. This simulation follows the second case of long-term behaviour.

ness on a plateau before equilibrating, unlike in the base case model. Additionally, we see that the longer the plateau phase, the more constant the substratum degradation rate. We see that as the threshold concentration increases, the biofilm thickness reaches a smaller maximum thickness, takes longer to equilibrate, and has a longer plateau phase, as described above. The increase in time to equilibrium is due to the degradation of the substratum. We see that increasing the threshold concentration decreases the rate of substratum degradation. Therefore, the biofilm degrades the substratum for longer. Furthermore, we can see that these simulations all follow the long-term behaviour described by case 2 ; see Figure 4.2 , i.e. $\lambda^{*}$ exists and $\lambda^{*}<\tilde{\lambda}$, since the final biofilm thickness is positive and the biofilm thickness achieved a maximum. Lastly, as shown in the analysis, all final biofilm thicknesses are the same as in the base case model.

\subsection{Effects of the biofilm/substratum yield coefficient}

In the third simulation, we investigate how the biofilm/substratum yield coefficient affects the growth of the biofilm and the degradation of the substratum in the first case of longterm behaviour. In these simulations, we set the threshold concentration to $\tilde{c}=0.002$ and investigate biofilm/substratum yield coefficients in the range of 0.1 to 0.75 . We also include the base case biofilm model (17). The simulation results are given in Figure 5.4.

We see that an increase in the biofilm/substratum yield coefficient increases the growth rate of the biofilm and the final substratum thickness. The increase in the final substratum thickness is due to the increased biofilm thickness, since an increase in the biofilm thickness, decreases the substratum degradation rate. We also see that the substratum completely degrades in this setting when the biofilm/substratum yield coefficient $A=0.1$. This still follows case 1 of long-term behaviour since that case does not exclude this possibility. Therefore, these simulations follow the first case of long-term behaviour; see Figure 4.1, i.e. $\lambda^{*}$ exists and $\lambda^{*}>\tilde{\lambda}$, since the substratum thicknesses are equilibrating at non-negative values and the biofilm growth is monotonic. Lastly, as shown in the analysis, all final biofilm thicknesses are the same as in the base case model. 

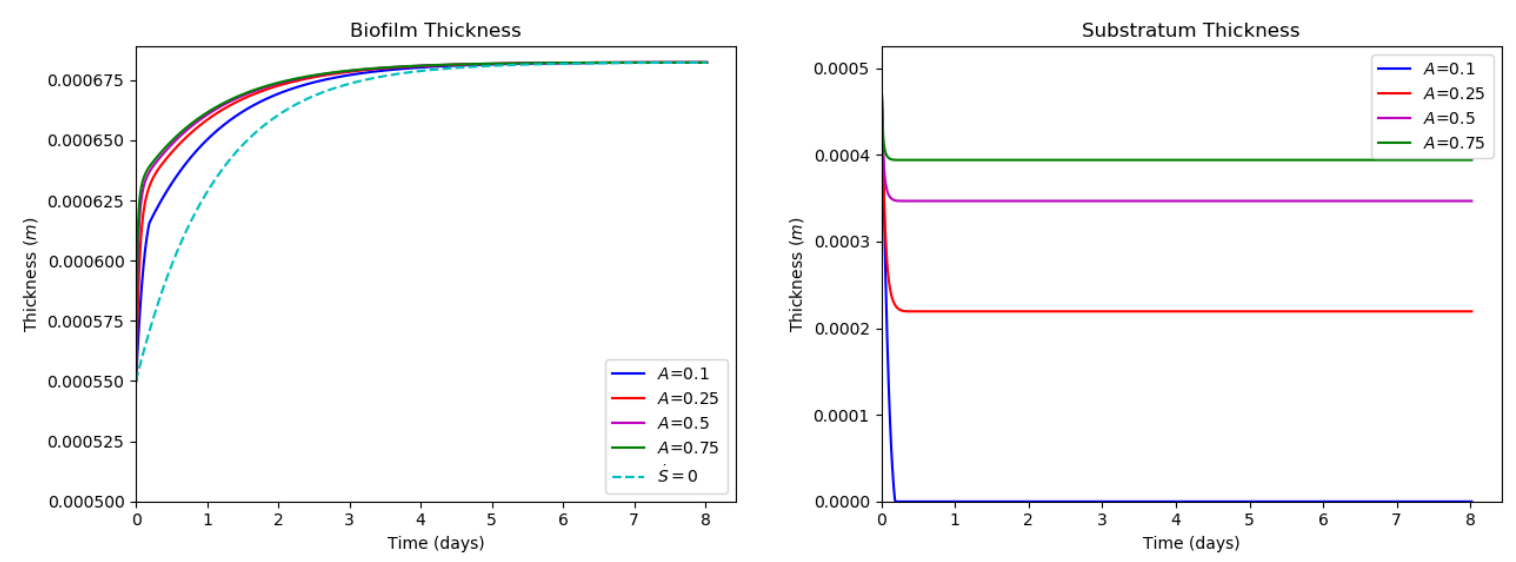

Figure 5.4: Comparison of the full system (5) and the base model (17) (dashed line), with varying biofilm/substratum yield coefficients $A$. Each simulation uses the parameter values summarized in Table 1 with $\tilde{c}=0.002$ and $A=\{0.1,0.25,0.5,0.75\}$. The biofilm thickness $\lambda$ and substratum thickness $S$ are given on the left and right, respectively. This simulation follows the first case of long-term behaviour.
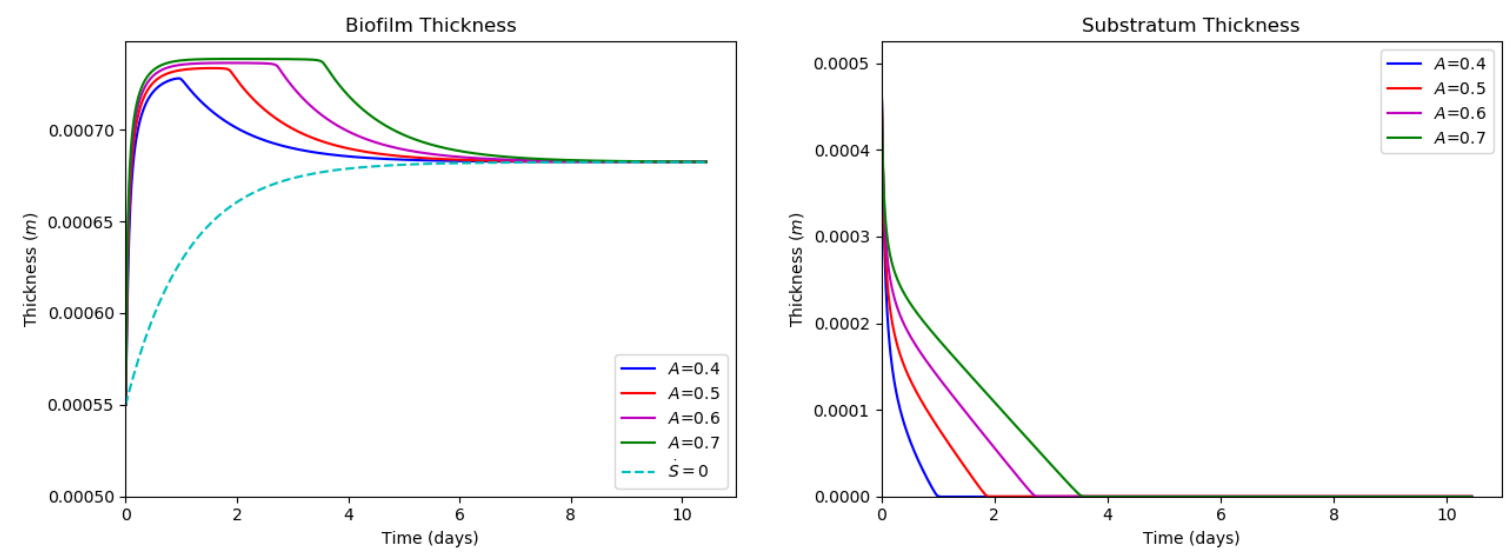

Figure 5.5: Comparison of the full system (5) and the base model (17) (dashed line), with varying biofilm/substratum yield coefficients $A$. Each simulation uses the parameter values summarized in Table 1 with $\tilde{c}=0.0002$ and $A=\{0.4,0.5,0.6,0.7\}$. The biofilm thickness $\lambda$ and substratum thickness $S$ are given on the left and right, respectively. This simulation follows the second case of long-term behaviour.

In the last simulation, we investigate how the biofilm/substratum yield coefficient affects the growth of the biofilm and the degradation of the substratum when we are in the second case of long-term behaviour. In these simulations, we set the threshold concentration to $\tilde{c}=0.0002$ and investigate biofilm/substratum yield coefficient values in the range of 0.4 to 0.7 . Moreover, we compare these simulations to the base case biofilm model (17). The simulation results are reported in Figure 5.5.

We see that the biofilm thicknesses in the full model reach a maximum thickness on a plateau before equilibrating, unlike in the base case model. Additionally, we see that the longer the plateau phase, the more constant the substratum degradation rate. We see that increasing the biofilm/substratum yield coefficient increases the maximum 
thickness achieved by the biofilm along with the time to equilibrium and the length of the plateau phase. The increase in the time to equilibrium is due to the degradation of the substratum. As the value of the biofilm/substratum yield coefficient increases, the rate of substratum degradation decreases, and thus, the biofilm degrades the substratum for longer. Furthermore, we can see that these simulations all follow the second case of long-term behaviour; see Figure 4.2, i.e. $\lambda^{*}$ exists and $\lambda^{*}<\tilde{\lambda}$, since the final biofilm thickness is positive and the biofilm thickness achieved a maximum. Lastly, as shown in the analysis, all final biofilm thicknesses are the same as in the base case model.

\section{Conclusion}

In this paper, we studied a mathematical model of biofilm growth on a degradable substratum, which is an extension of a simpler model (17) where the biofilm was growing on an inert substratum. The purpose was to study the effect of substratum degradation on such models that permit this. We started with the simple case where substratum degradation occurs if and only if the bacteria at the biofilm-substratum interface layer are active. With this model, we were interested in investigating whether or not such an extension would yield different system behaviour. We found that the equilibria of the system and the stability conditions were the same as in the simpler model, i.e. the extension with substratum degradation produced the same long-term behaviour. However, the time evolution of the system is different. We saw that the biofilm thickness does not necessarily grow monotonically, which is different from the growth of the biofilm in the base case model. Therefore, the addition of substratum degradation in systems that satisfy our model assumptions is necessary to describe system behaviour accurately.

The non-monotonic growth of the biofilm thickness in certain simulations leads to interesting consequences for systems satisfying our model assumptions. For example, suppose we are modelling a reactor where there is a biofilm growing, and these biofilms degrade the substratum based on our model assumptions. If we are concerned with substrate removal inside the reactor, then if we used the simpler biofilm growth model (17) instead of our model (5) we would see an underestimate in the substrate removal. The underestimate of the substrate removal would be caused by the underprediction of the total flux of substrate into the biofilm. Which we can see by considering Figure 5.3 and that the flux function $J\left(\lambda ; C_{\infty}\right)$ is increasing in $\lambda$. Therefore, for systems satisfying our model assumptions, it is necessary to include the effects of substratum degradation.

When considering our biofilm growth model, the only way to change the biofilm thickness in the long-term is to scrape off part of the biofilm. In the context of our model, this would be the increase of the detachment rate $d(\lambda)$. As we can see from our results in Sections 4.1 and 4.2, specifically corollaries 4.10 and 4.14 respectively, an increase in the detachment rate can cause a change in long-term behaviour, namely from case 1 to case 2. This change in long-term behaviour is a reflection of the change in the inactive layer of the biofilm. A thicker biofilm means less dissolved substrate for the bacteria at the base of the biofilm, which means a larger inactive layer and, in the context of our model, no substratum degradation. This system behaviour reflects biological phenomenon as we expect a thinner biofilm to have a smaller inactive layer.

While the model is simplified, it still provides beneficial first insight into the effects of substratum degradation on biofilm growth. The simplicity of the model allowed for detailed analytical results from existence and uniqueness to equilibria and stability analysis. 
However, an investigation into different degradation kinetics could prove very useful to our understanding of the interactions between biofilms and the substratum. For specific applications, one would likely need to adapt the substratum degradation kinetics to fit their system best, but what we have shown is that the addition of substratum degradation can have a significant impact on the dynamics of these systems. Thus, when applicable, substratum degradation should be implemented in such models.

There are plenty of biofilm growth models in the literature that investigates biofilm formation/growth on inert substratum in more complex settings. For example, models that account for variable bulk substrate concentration and suspended bacterial flocs $[5,9]$, or models which assume a multi-species biofilm as in [14]. Therefore, a further study of biofilm growth on degradable substratum in various settings would also be beneficial to the understanding of biofilm/substratum interactions.

\section{References}

[1] F. AbBAs And H. J. EBERL, Investigation of the role of mesoscale detachment rate expressions in a macroscale model of a porous medium biofilm reactor, Int. J. Biomath. Biostat., 2 (2013), pp. $123-143$

[2] F. AbBas, R. Sudarsan, And H. J. EBerL, Longtime behaviour of one-dimensional biofilm models with shear dependent detachment rates, Math. Biosci. Eng., 9 (2012), pp. 215-239

[3] U. M. Ascher, R. M. M. MattheiJ, And R. D. Russell, Numerical solution of boundary value problems for ordinary differential equations, SIAM, Philadelphia, PA, 1995

[4] J. R. Dormand And P. J. PRInce, A family of embedded Runge-Kutta formulae, J. Comput. Appl. Math., 6 (1980), pp. 19-26

[5] H. J. Gaebler AND H. J. EBerL, A simple model of biofilm growth in a porous medium that accounts for detachment and attachment of suspended biomass and their contribution to substrate degradation, European J. Appl. Math., 29 (2018), pp. 1110-1140

[6] J. Kierzenka And L. F. Shampine, A BVP solver based on residual control and the Maltab PSE, ACM Trans. Math. Software, 27 (2001), pp. 299-316

[7] I. Klapper, Productivity and equilibrium in simple biofilm models, Bull. Math. Biol., 74 (2012), pp. $2917-2934$

[8] Z. Lewandowski And H. Beyenal, Fundamentals of Biofilm Research, CBC Press, Boca Raton, FL, 2007

[9] A. MAS̆IĆ, H. J. EBerL, Persistence in a single species CSTR model with suspended flocs and wall attached biofilms, Bull. Math. Biol., 74 (2012), pp. 1001-1026

[10] Y. G. Maksimova, Microbial biofilms in biotechnological processes, Appl. Biochem. Micro., 50 (2014), pp. $750-760$

[11] P. Virtanen, R. Gommers, T. E. Oliphant, M. Haberland, T. Reddy, D. Cournapeau, E. Burovski, P. Peterson, W. Weckesser, J. Bright, S. J. van der Walt, M. Brett, J. Wilson, K. J. Millman, N. Mayorov, A. R. J. Nelson, E. Jones, R. Kern, E. Larson, C. Carey, İ. Polat, Y. Feng, E. W. Moore, J. VanderPlas, D. Laxalde, J. Perktold, R. Cimrman, I. Henriksen, E. A. Quintero, C. R. Harris, A. M. Archibald, A. H. Ribeiro, F. Pedregosa, P. van Mulbregt, And SciPy 1.0 Contributors, SciPy 1.0: fundamental algorithms for scientific computing in Python, Nat. Methods, 17 (2020), pp. 261-272

[12] W. Walter and R. Thompson, Ordinary Differential Equations, Springer, New York, NY, 1998

[13] O. Wanner, H. J. Eberl, E. Morgenroth, D. R. Noguera, C. Picioreanu, B. E. Rittmann, And M. VAn Loosdrecht, Mathematical Modelling of Biofilms, IWA Publishing, London, England, 2006

[14] O. Wanner And W. Gujer, A multispecies biofilm model, Biotechnol. Bioeng., 28 (1986), pp. 314328 